\title{
The research of road extraction from high-resolution remote sensing image based on optimized watershed algorithm
}

\author{
Hongyue Cai, Guoqing Yao \\ School of Information Engineering \\ China University of Geosciences (Beijing) \\ Beijing, China \\ redmoon1126dida@163.com
}

\begin{abstract}
Recently the major methods of extracting road from remote sensing image are manually. It becomes a hotpot to realize automatic information extraction of road according to the feature of high-resolution image and the characteristics of road. In this paper, an improved approach for road extraction based on Watershed segmentation is proposed according to theories of object-oriented and mathematical morphology. The image is first processed by improved Watershed segmentation to acquire feature-units after preprocessing. Then object-oriented method is used to analyze road feature-units in order to optimize the road extraction results. Finally, after binary image processing, the incomplete results can be removed and corrected by using mathematical morphological transformation. At last this paper gives the result and analysis of the experiment sample.
\end{abstract}

Index Terms-Road extraction, high-resolution remote sensing image, watershed, object-oriented method, mathematical morphology.

\section{INTRODUCTION}

With the commercialization of remote sensing application, high resolution remote sensing images catch our attentions. Roads, the main part of the modern transportation system, play an important part in economic activities. Roads are essential geographic data for cartography, rout analysis and emergency. But nowadays, roads especially in urban areas are still extracted manually, which does not meet the demand of database updating. Automatic procedures are desirable for road extraction from remote sensing images. In recent year, the approaches of road extraction from high-resolution remote sensing images have become a focus.

There are many different approaches for road extraction from high-resolution remote sensing imagery. At present, template matching and snakes model are methods for roads extraction based on spectrum feature [1-2]. But for highresolution remotely sensed images, the phenomenon that the same thing having different spectrums and the same spectrum belongs to different things is very obvious, these traditional methods could not solve the problem. Texture-based method and hierarchical cluster have been taken context into consideration [3], but the typical characteristics in high- resolution images such as geometry and spatial relationship haven't been fully used. Artificial intelligence and computer vision are direction for road extraction in recent year, but they are still in the study [4-5].

The purpose of this project is to develop an algorithm to facilitate automated road extraction from high-resolution satellite remote sensing images. A QuickBird satellite panchromatic image with resolution $0.6 \mathrm{~m}-0.7 \mathrm{~m}$ is used in this study.

\section{Methodlogy And TeCHNICAL Route}

This paper proposes an optimized watered algorithm to improve road extraction in suburban areas based on problems of extraction in high-resolution remote sensing. Watershed algorithm is a method comes from geography, which transforms the image into closed-connected segmentations based on topology characteristic of images. To avoid the oversegmentation of this algorithm, this paper takes some feasible measures to improve. After image segmentation, the image will be segmented into multiple sections. Each section can be seen as a feature-unit. Feature-units are the basic unit of in objectoriented analysis, which have the characteristic of geometry, topology and spatial relationship, etc. The characteristics of different objects can be used to refine the roads broken up by zebra stripes or shadow, whereas analysis based on pixels cannot achieve. Operations in mathematical morphology algorithm are also used to make further improvement for extraction results. At last we give the experiments analysis to show the efficiency of proposed approach to road extraction.

The work flow of the proposed method includes image preprocessing, image segmentation, feature extraction and post-processing, see in Fig.1.

\section{A. Image preprocessing}

Because of the context of experiment images is relatively dark, histogram equalization is firstly used to enhance the difference between the roads and the surroundings. Then median filter is applied to remove impulse noise at same time keeping the edge of the image, in order to reduce over segmentation and reduce jumping edges. 

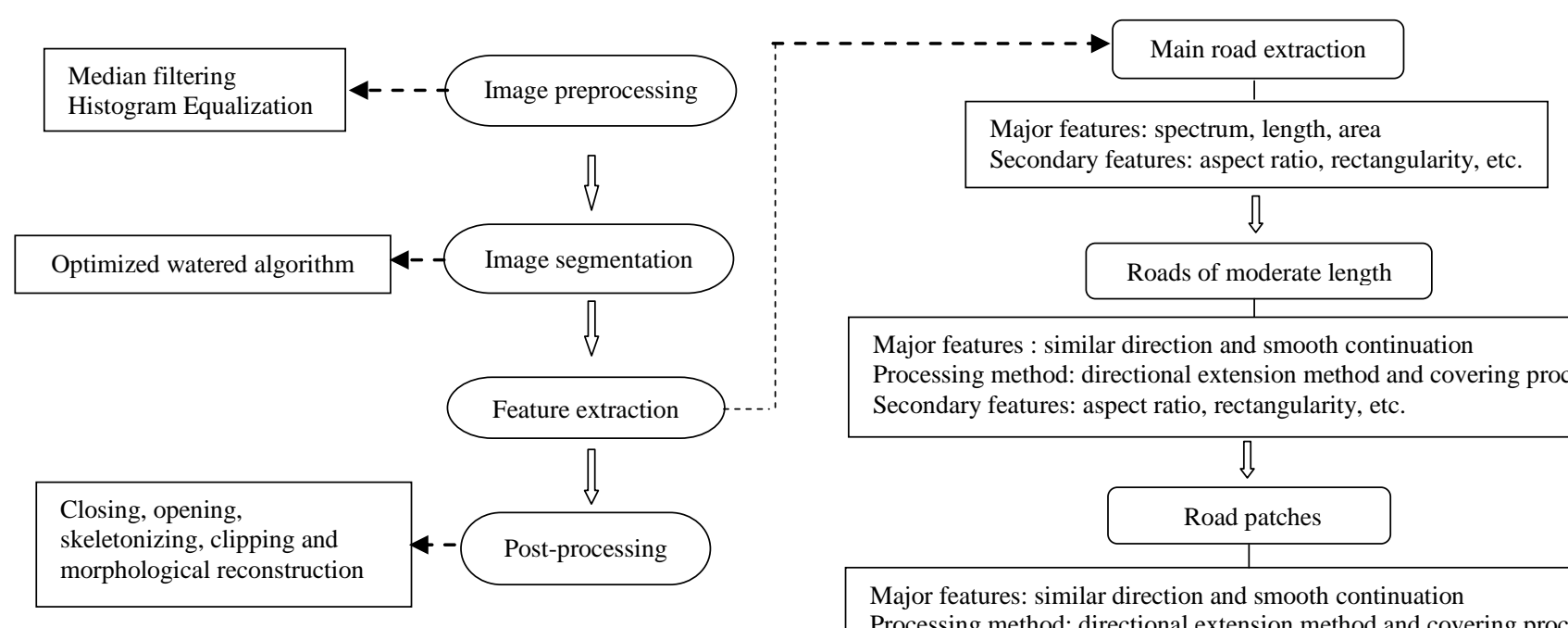

Major features : similar direction and smooth continuation Processing method: directional extension method and covering processing Secondary features: aspect ratio, rectangularity, etc.

Fig. 1. Flow chart of road extraction.

\section{B. Image segmentation}

In this paper, we implement image segmentation by multilevel immersion watershed algorithm based on region-growth [6]. By selecting appropriate local homogeneity threshold, removing small basin, merging small patches and region merging to achieve the accurate results and save time for later processing.

In the gradient image, the pixel whose value less than the local homogeneity threshold can be seen as the local minimum of each basin. Each connected region of local minimum pixels form an initial basin. With the local homogeneity threshold increases, connected basin under the water level of the threshold will gradually merge. Figure 2 shows the relationship between local homogeneity threshold and region number. In order to achieve better segmentation results, the threshold which makes basin increasing balance with merging can be selected according to the segmentation results (that is, the values between 5 and 8 in Fig. 2 are preferred values).

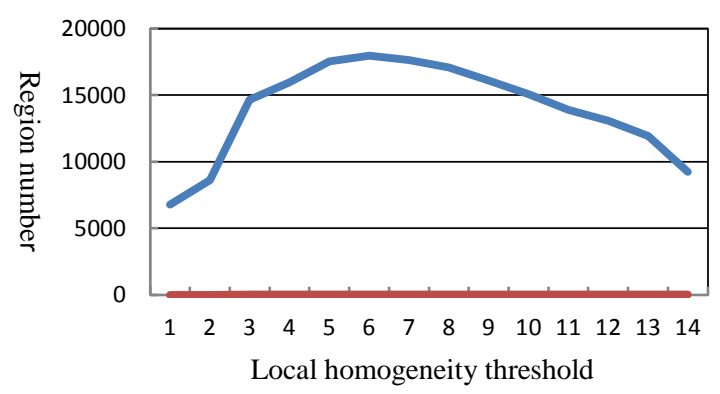

Fig. 2. Relationship between local homogeneity threshold and region number.

Region merging is the classic algorithm to reduce oversegmentation. In this paper, we use minimum area (MinRegion) and minimum average spectrum difference (MaxGray_R) to control the region merging process. That is, if one region whose area less than MInRegion and average spectrum difference between with its neighborings less than MaxGray_R, the region will be merged.

Because of region merging having a high computational cost, meaningless small patches are first removed in order to save time for later processing. The merging principle is as follows: for each pixel (take the red pixel in Fig.3 for example), if the area of patch (shown by the shaded section in Fig.3) where the pixel located in less than the minimum area of all the patches (MinPatch), then finding the most similarest neighboring for this patch according to minimum spectrum difference in scaning region. The scaning region has a radius of $\sqrt{\text { MiniPatch }}$ in horizontal and vertical direction ( shown by the green section in Fig.3). If the spectrum difference between the similarest patch and the patch where this pixel located in less than the maximum spectrum difference $\left(M a x G r a y \_P\right)$, merging them into one patch.

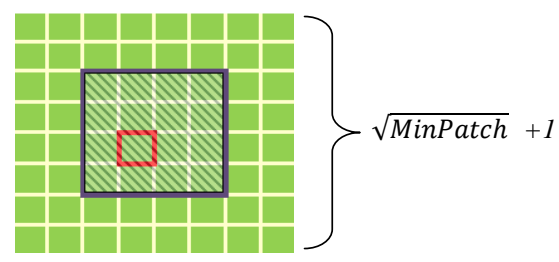

Fig. 3. Principle of merging small patches.

\section{Road feature extraction}

The image is transformed into different objects of feature-unit after watershed segmentation. Roads are crossgroup network having characteristics of larger length, larger aspect ratio and having context objects such trees and shadow which are different from other objects. Object-oriented method can take full advantage of the spectral, geometric, topology, context feature of roads to optimize the road extraction. The road extraction is refined by feature such as 
length, aspect ratio, rectangularity, directional extension method and covering processing.

\section{1) Object-oriented}

In Object-oriented, calculating the length, aspect ratio and rectangularity are all based on calculating minimum bounding rectangle of per-parcel. Minimum bounding rectangle calculated by rotating per-parcel is shown in Fig.4.

Calculate the area of minimum bounding rectangle at different angles while rotating the feature-unit with the center of minimum bounding rectangle. Update the information of minimum area of minimum bounding rectangle each times, and record the rotating angel as $\theta_{0}$. In the end, after finish rotating, rotate the minimum bounding rectangle of minimum area with angle $-\theta_{0}$, and then get the minimum bounding rectangle of the initial feature-unit. In this paper, we calculate the minimum bounding rectangle by rotating parcel for 180 times, with angel of $1^{\circ}$ for each time.

At the same time, there are many intersections in road feature-unit which are similar with the buildings feature-unit, but the rectangularity of building is larger. Rectangularity, the ratio between the area of feature-unit and the area of minimum bounding rectangle is another feature to identify the roads from buildings.

\section{2) directional extension method}

The most distinguishing characteristic of road feature-units in image is having larger length according to amounts of experiments. Most main roads can be extracted by length feature, but short road feature-units due to noise do not have obvious differences between other objects in spectrum and shape. Roads are network generally with small curvature, so there is evidence that a small road feature-unit belong to the main road, such as similar direction and smooth continuation. This paper, we use directional extension method presented by Luo Q Z, Yin Q and Kuang D B to extract small road featureunits from noise [7].

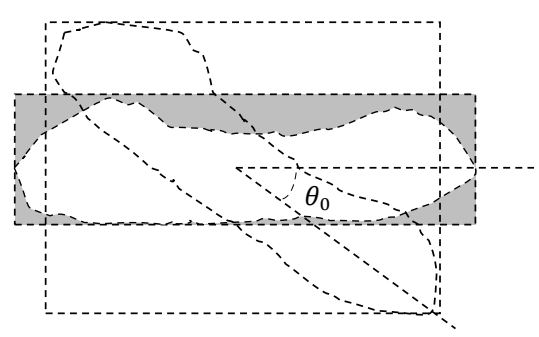

Fig. 4. Minimum bounding rectangle calculated by rotating per-parcel.

\section{3) Covering processing}

The disappearance of the complete road edge is caused by context objects such as buildings and shadow near the roads. Zhu X L proposed a method to resolve this problem based on the same edge between roads and coverings: If ratio between the length of same edge and the length of the whole edge of the covering (Ratio) larger than 0.5, take the covering as roads [8]. The rest coverings can be done in the same manner until all the coverings has been processed (as shown in Fig.5).

This method can fill the disappearance of roads, but it takes the whole covering as the road information which still affects the complete of roads. In this paper, we use the method similar to minimum bounding rectangle to optimize the covering processing has been mentioned.

All the pixels of the same edge of the road parcel and adjacent covering is firstly detected, and then calculate the minimum bounding rectangle of these pixels, take the minimum bounding rectangle as roads information to fill the extracted roads. This proposed method has good effect when the curvature is not large. At the same time all the covered roads can be refined whatever Ratio is.

\section{4) Post-processing}

The roads are mainly extracted by object-oriented method, and then implement binary mathematical morphology operators to improve the road information such as filling the hole and linking the gaps in road network. The basic principle of mathematical morphology is detecting and extracting the geometry according to the structural element to identify and analyze image. Its application fields include edge detecting, skeletonizing, morphological analysis, image reconstruction and so on. In this paper, closing, opening, skeletonizing, clipping and morphological reconstruction are used to refined road information.

The holes caused by rotating process due to the discrete characteristic of raster can be filled by closing operation, using structural element of 3 pixel $\times 3$ pixel with each value of 1 . In addition, closing operation also can link gaps in road network.

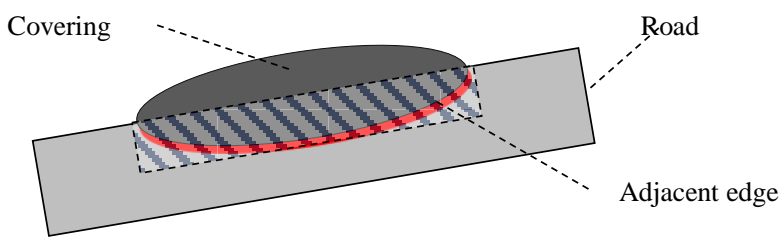

Fig. 5. Principle of covering processing.

As to other isolated objects or objects stuck together with roads, opening (erosion first and then dilation) operator is fist used to separate the objects, and then removed the small region after calculating area of each region. The larger holes of road caused by vehicles can also be filled with the similar method. In the other way, all-dimensional detecting and morphological reconstruction with appropriate length and direction are means of removing other objects further [9-10]. Clipping algorithm and short segments removing can delete redundant parasitic components in order to optimize the results of directional extension method after automatic vectorization by Hilditch skeleton algorithm[11-12].

\section{RESULTS AND ANALYSIS}

Here we use select a urban image of QuickBird as the experiment images with spatial resolution of $0.6 \mathrm{~m}-0.7 \mathrm{~m}$, see Fig.6.

In road extraction experiment, extracting main roads is an important step, which further processing will rely on. In this step, the results should be optimized as much as possible. In this experiment, road feature-units of moderate length are classified into two parts: length larger than 25 pixels and less than 25 pixels. Road feature-units of moderate length whose 


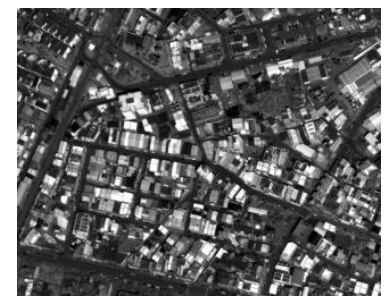

(a)

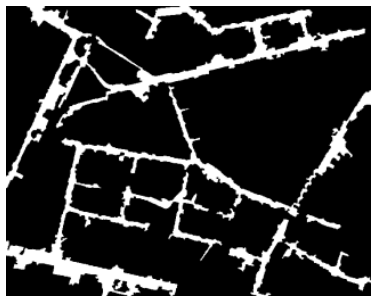

(b)
Fig. 6. Experiment of road extraction. (a)Initial image. (b)Result of the road extraction.

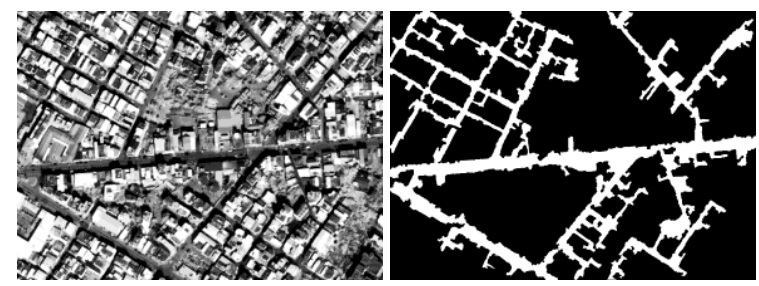

Fig. 7. Another experiment of road extraction. (a)Initial image. (b)Result of the road extraction.

length larger than 25 which have been extracted in the first step(object- oriented) because of the spectrum difference. So these road feature-units are prior considered. Road featureunits whose length less than 25 are similar with buildings, so shape feature and rectangularity are additional feature to complete roads extraction.

In road extraction experiment, extracting main roads is an important step, which further processing will rely on. In this step, the results should be optimized as much as possible. In this experiment, road feature-units of moderate length are classified into two parts: length larger than 25 pixels and less than 25 pixels. Roads feature-units of moderate length whose length larger than 25 does extracted in the first step(objectoriented) because of the spectral difference. So these roads feature-units are prior considered. Road feature-units whose length less than 25 are similar with buildings, so shape factor[] and rectangularity are additional feature to complete roads extraction.

From the road extraction result in Fig.6, most roads are extracted completely except some branchs of short length and few road edges are lost. Another experiment of road extraction is shown in Fig.7.

\section{EVAluATE}

This paper presents a technical route of road information extraction from high resolution remote sensing image and the results show a good effect even the surroundings is complicated. Improved watered algorithm is a good way of segmentation, reducing over segmentation. Object- oriented fully use of the feature such as geometry, topology to improve the result of road extraction. Binary morphology not only the assistant means for steps have mentioned above, but also improve the roads result further.
But the proposed method still has something needs improved and researching. Because of object-oriented analysis relies on results of image segmentation.This paper does not take texture into consider, the method based on one band of reducing over segmentation is simple, and the efficiency of region merging needs improved. SVM classification and prior knowledge also have not been applied. How to extracting roads more intelligently and automaticly by technique from different fields and evaluation for extraction results are what need further researching.

\section{REFERENCES}

[1] Hu X Y, Zhang Z X, Zhang J Q, "Semiautomatic extraction of linear object from aerial image," Journal of Image and Graphics, vol. 7, no. 2, pp. 137-140, 2002.

[2] Ding M L, Li G Y, Zhang Q F, “Application of road extraction in satellite images based on snakes model," Computer Technology and Development, vol. 1, no. 1, pp. 71-73, 2010.

[3] Zhang R, Zhang J X, Li H T, "Semi-automatic extraction of ribbon roads from high resolution remotely sensed imagery based on angular texture signature and profile match," Journal of Remote Sensing, vol. 12, no. 2, pp. 224-232, 2008.

[4] Lin L Q, Xiao J, "Combined prior knowledge and image characteristics for lane detection method," Computer Engineering and Applications, vol. 47, no.33, pp. 236-239, 2011.

[5] Ning Y H, Lei X Q, Wang G X, Li S Q, "Road extraction from high-resolution remote sensing images based on DempsterShafer evidence theory and fusion shape features, " Journal of Image and Graphics, vol. 16, no. 12, pp. 2183-2190, 2011.

[6] Pan T T, Li C Z, "Road extraction method of satellite image based on Region-Growth Watersheds Algorithm," Computer Engineering and Design, vol. 29, no. 19, pp. 4987-5013,2008.

[7] Luo Q Z, Yin Q, Kuang D B, "Research on extracting road based on its spectral feature and shape feature," Remote Sensing Technology and Application, vol. 22, no. 22, pp. 339-344, 2007.

[8] Zhu X L, Wu Q Y, "Study on the method of extracting city roads from the high resolution remote sensing image," Resources Environment \& Engineering, vol. 23, no. 3, pp. 296299, 2009.

[9] Vincent L, "Morphological grayscale reconstruction in image analysis: Applications and Efficient Algorithms," IEEE Tranns. Image Process., vol.2, no.2, pp. 176-201,1993.

[10] Shen S H, Song Y, Wan Y C, Liu S F,“An approach for automatic road extraction in dense urban area from highresolution multispectral satellite imagery," Remote Sensing Information, no. 8, pp. 73-77, 2007.

[11] Gaetano R, Zerubia J, Scarpa G, "Morphological road segmentation in urban areas from high resolution satellite images," Digital Signal Processing (DSP), Corfu Greece, pp. 18, 2011.

[12] Zhu C, Shi W, Pesaresi M, "The recognition of road network from high-resolution satellite remotely sensed data using image morphological characteristic," International Journal of Remote Sensing, vol. 26,no. 24,pp. 5493-5508, 2005. 